\title{
Assessment of the Cutting Performance of a Robot Mower Using Custom Built Software
}

\author{
Luisa Martelloni ${ }^{1, *}$ (), Marco Fontanelli ${ }^{1}$, Stefano Pieri ${ }^{2}$, Christian Frasconi ${ }^{1}$, Lisa Caturegli ${ }^{1}{ }^{\circledR}$, \\ Monica Gaetani ${ }^{1}$, Nicola Grossi ${ }^{1}{ }^{\mathbb{D}}$, Simone Magni ${ }^{1}{ }^{\mathbb{D}}$, Michel Pirchio ${ }^{1}$, Michele Raffaelli ${ }^{1}$, \\ Marco Volterrani ${ }^{1}$ and Andrea Peruzzi ${ }^{1}$ \\ 1 Department of Agriculture, Food and Environment, University of Pisa. Via del Borghetto 80, 56124 Pisa, \\ Italy; marco.fontanelli@unipi.it (M.F.); christian.frasconi@unipi.it (C.F.); lisa.caturegli@gmail.com (L.C.); \\ monica.gaetani@unipi.it (M.G.); nicola.grossi@unipi.it (N.G.); simone.magni@unipi.it (S.M.); \\ michel.pirchio@for.unipi.it (M.P.); michele.raffaelli@unipi.it (M.R.); marco.volterrani@unipi.it (M.V.); \\ andrea.peruzzi@unipi.it (A.P.) \\ 2 Qprel ${ }^{\circledR}$ srl. Via Fiorentina 578/D, 51100 Pistoia, Italy; stefano@qprel.com \\ * Correspondence: lmartelloni@agr.unipi.it; Tel.: +39-050-2218-966
}

Received: 22 March 2019; Accepted: 1 May 2019; Published: 6 May 2019

\begin{abstract}
Before the introduction of positioning technologies in agriculture practices such as global navigation satellite systems (GNSS), data collection and management were time-consuming and labor-intensive tasks. Today, due to the introduction of advanced technologies, precise information on the performance of agricultural machines, and smaller autonomous vehicles such as robot mowers, can be collected in a relatively short time. The aim of this work was to track the performance of a robot mower in various turfgrass areas of an equal number of square meters but with four different shapes by using real-time kinematic (RTK)-GNSS devices, and to easily extract data by a custom built software capable of calculating the distance travelled by the robot mower, the forward speed, the cutting area, and the number of intersections of the trajectories. These data were then analyzed in order to provide useful functioning information for manufacturers, entrepreneurs, and practitioners. The path planning of the robot mower was random and the turfgrass area for each of the four shapes was $135 \mathrm{~m}^{2}$ without obstacles. The distance travelled by the robot mower, the mean forward speed, and the intersections of the trajectories were affected by the interaction between the time of cutting and the shape of the turfgrass. For all the different shapes, the whole turfgrass area was completely cut after two hours of mowing. The cutting efficiency decreased by increasing the time, as a consequence of the increase in overlaps. After 75 minutes of cutting, the efficiency was about $35 \%$ in all the turfgrass areas shapes, thus indicating a high level of overlapping.
\end{abstract}

Keywords: GNSS; lawnmower; localization; mowing; random coverage path planning; trajectory tracking; turfgrass

\section{Introduction}

Before the introduction of positioning technologies, data collection and management in agriculture was time-consuming and labor-intensive, however technologies today enable more precision field data to be acquired [1]. Global navigation satellite systems (GNSS) devices are used to monitor agricultural operations and to acquire field data [2,3]. Such data then need to be stored, processed, analyzed, and interpreted using software. Results of the data analysis can be used by manufacturers, entrepreneurs, and practitioners to facilitate their decision-making, reduce costs, and improve the quality of the product [4]. The real-time kinematic (RTK) tool is widely used in high-precision positioning and navigation applications, and can also be used in automated driving use cases [5]. 
Trajectory tracking systems were studied for planning the path that an autonomous robot has to follow within the field for conducting a specific operation [6-9]. Mohamed et al. [6] generated an optimal free-obstacle path of a robotic vehicle that moved from a starting point to the goal location. Kassaeiyan et al. [9] applied their developed trajectory tracking systems to a real-world experimental implementation. Indeed, the most important problem among the trajectory tracking is the applicability in real-world applications [9].

The RTK-GNSS devices are used to record vehicle movements [10-13]. For under open sky road driving, the RTK solution generated accurate and precise vehicle movement trajectories sufficient for extracting vehicle lane position, speed, acceleration, and deceleration [10]. Huang et al. [11] equipped an autonomous robot with a GNSS antenna and receiver in order to control the position of the robot with respect to the target path. El-Mowafy and Kubo [12] integrated RTK-GNSS, doppler-based positioning, and positioning using a low-cost inertial measurement unit (IMU) with a car speedometer to achieve continuous positioning of an autonomous vehicle. Sun et al. [13] used the RTK-GNSS to pursue precise vehicle movement trajectory in an urban residential area of Australia.

Different methods than RTK-GNSS for path tracking are used preferably in GNSS-denied environments because these methods had a lower accuracy (e.g., IMU), are more costly and of hard installation (e.g., LiDAR odometry), and of difficult calibration (camera based systems) [14-17].

Coverage path planning involves determining a path that passes over all points of an area of interest while avoiding obstacles [18]. This task is integral to robotic mowers [19]. The majority of current commercial robot mowers move randomly by travelling along straight lines and making turns at the perimeter (boundary wire) of the cutting area or any other obstacle [20,21]. The main advantage of random path planning is that no complex sensors for localization or expensive computational resources are needed [18].

Path planning and trajectory tracking control for robot mowers based on RTK-GPS were studied in order to plan a pre-planned linear path that the robot has to follow [22]. High technology and highly expensive robot mowers, such as the L400 Elite model manufactured by Zucchetti Centro Sistemi Spa (Arezzo, Italy), are equipped with a differential global position system (D-GPS) guidance system that enables the cutting path to be pre-planned [23]. The use of this technology reduces the operating costs (energy and time) [9]. However, GPS devices may function inaccurately due to the interference of buildings and tree canopies in residential turf areas [14,24]. The objective of path planning for mobile robots is to find an optimal collision-free path from a starting point to a target point [25], however the high-cost of the technology used for these systems make the real and systematic application in the field difficult. Moreover, the signals of GNSS devices are blocked frequently in challenging environments, and the discontinuous carrier phases seriously affect the application of GNSS precise positioning reducing the adoption of these systems [26]. Robot mowers that move randomly are indeed nowadays largely used.

To the best of our knowledge there has been no research studying the random trajectories followed by commercial robot mowers, with the aim to improve the cutting efficiency of a robot that moves randomly whose sales are currently increasing. The objective of this work was to track the performance of a robot mower in different shapes of turfgrass areas using real-time kinematic (RTK)-GNSS devices, and to easily extract useful data using a custom built software capable of calculating the distance travelled by the robot mower, the forward speed, the cutting area, and the number of intersections of the trajectories. For each of these variables, the used datasets included data extracted from the software every $15 \mathrm{~min}$ for a total time of two hours, in order to have nine time points for four replicates of the experiment in each turfgrass area shape. These data were then analyzed in order to provide useful information for manufacturers, entrepreneurs, and practitioners. 


\section{Materials and Methods}

\subsection{The Robot Mower Tracking Data System}

A system to track the performance of a robot mower within a turfgrass delimited by the boundary wire was designed and built at the request of the University of Pisa. The system was composed of a remote sensing system for the acquisition of positioning and time data, along with custom-built software to calculate, display, and extract these data. The system tracks the trajectories of the robot mower and calculates the percentage of cut turfgrass area as a function of time, together with other operative data relative to the performance of the robot mower.

\subsubsection{The Remote Sensing System to Acquire Data}

The remote sensing system was constituted by two Emlid Reach RTK devices [27] mounted in custom-built cases, one used as a base station and the other as a rover. The Reach RTK is a very small device, equipped with a real-time kinematic-global navigation satellite system (RTK-GNSS) receiver (ublox NEO-M8T series) [28] and an Intel ${ }^{\circledR}$ Edison module [29]. The Reach RTK is only a few millimeters larger than the Intel Edison module that it contains. The Intel@Edison module components are an Intel Atom dual core $500 \mathrm{MHz}$ Processor (with a serial port, a port with bus I2C, an in-circuit programming port, and connectivity for a SD card); 1 GB of memory DDR3 low power; 4 GB of memory EMMC; a USB 2.0 (both device and host); a Wi-Fi and Bluetooth Low Energy 4.0 module; and an on-chip integrated antenna.

Due to a custom Linux distribution, the Reach RTK device enables the RTK algorithm to be converged, and to manage the Wi-Fi and Bluetooth connections. The device has dual DF13 connectors and comes with all the required cables to connect to other devices. The antenna connector is MCX. The Reach RTK can both receive power from a USB, acting as a device, and a source power to the port, acting as a host [27]. The Reach RTK runs open-source RTK processing software called RTKLIB written by Takasu [30]. Previously a computer was required to run RTKLIB, but now all RTKLIB features are available directly on the Reach RTK device.

The case containing the rover was mounted on the robot mower and the case containing the base station was installed on the edge of the turfgrass (Figure 1). The inner components of the cases were reported in Figure 2. The rover was fed by a lithium battery (power bank $9000 \mathrm{mAh}$ ) with $24 \mathrm{~h}$ of battery life. The base station was fed by a USB cable, and a buffer battery was also mounted (power bank $6000 \mathrm{mAh}$ with five hours of battery life). The RTK algorithm precisely calculated the distance between the base and the rover. This distance was called the "baseline". The rover position was precisely determined relative to the base station position ( $\pm 7 \mathrm{~mm}$ on the horizontal plane). For this purpose the two Emlid Reach RTK devices [27] were used. They received signals from the GNSS and estimated their distance using the RTK algorithm implementation. The recorded data were downloaded from the Emlid Reach RTK devices [27]. Then the RTKLIB software framework was used to extract the final rover coordinates with the relative accuracy. The rover coordinates offset from the real location depended on the position accuracy of the base. The base station measured errors, and stationary it transmitted corrections to the rover. The rover and the base station communicated through a wireless network created by a common access point (NGB-418N v2, Zyxel) [31].

Both devices were managed by the web interface ReachView [32], which allows settings, analysis parameters, and the downloading of positioning and time data acquired. Data were recorded from the rover and the base, and were processed afterwards. RTKLIB version 2.4.3 was used to process data. The off-line processing of data enabled a position file (a formatted text file, .pos) to be created, which reported the relative positions (east, north, and up (ENU) components) between the rover and the base station (baseline), which represented the trajectories travelled by the robot mower in the turfgrass area. 


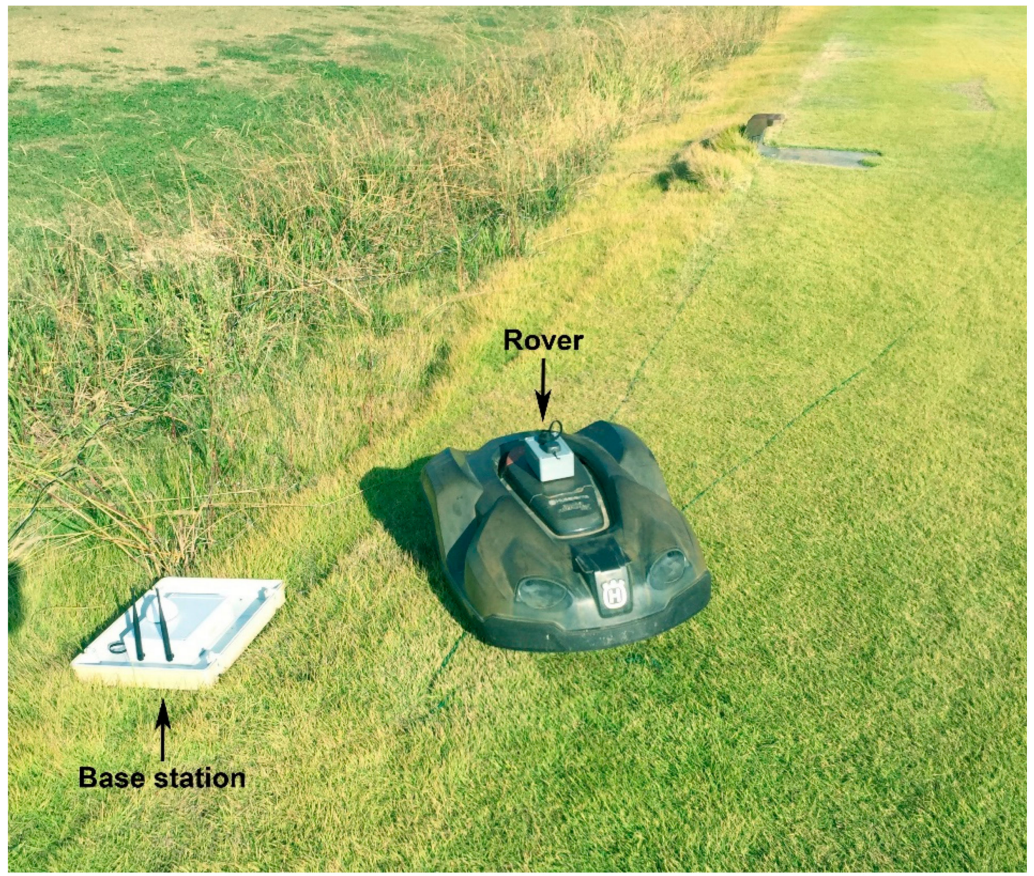

Figure 1. Custom-built cases containing the two Reach real-time kinematic (RTK) devices, one functioning as a base station and the other as the rover. The rover was mounted on the robot mower, and the base station was positioned at the edge of the turfgrass.

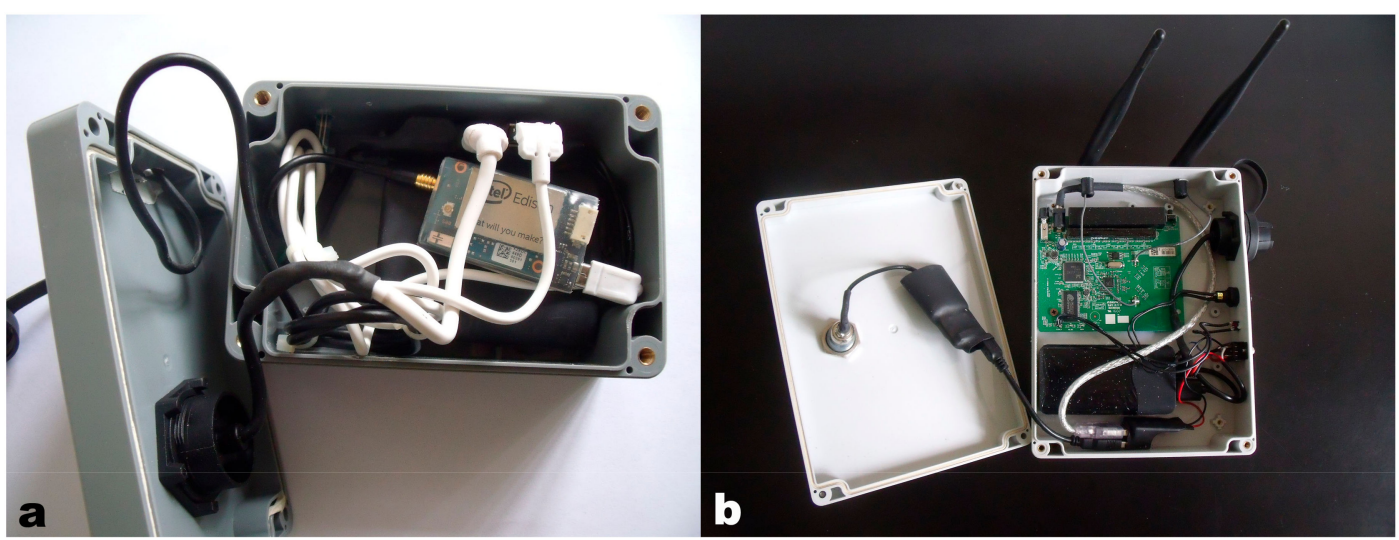

Figure 2. Inner components of the rover (a) and of the base station (b).

\subsubsection{New Software to Calculate, Display, and Extract Useful Data}

New software, called "Robot mower tracking data calculator", was designed and built by the Qprel $® s r l$ (Pistoia, Italy) [33] at the request of the University of Pisa, in order to extract data on the functioning of the robot mower. The function of the software was to allow an operator to easily get access to the operating data of the robot mower, and was developed mainly to calculate the area covered by the random trajectory of the robot mower with the passing of time.

The calculation algorithm designs the trajectories using the RTK coordinates recorded and the width of cut of the robot mower (which changes with the type of robot mower used), and from this design the percentage of coverage is calculated, corresponding to the cut area. The software simply colors by green the cut area following the robot trajectories and then calculates the ratio between the green area and the entire cutting area. The calculation algorithm and the graphics of the new software were developed using Visual Studio 2015 [34].

In addition to the cut area, the "Robot mower tracking data calculator" software processes the position file obtained with the RTKLIB software, and calculates every $5 \mathrm{~s}$ the mean forward speed of 
the robot mower, the intersections of the trajectories, the distance travelled, and the percentage area of the turfgrass cut. The software also shows other processed data such as the number of points recorded, total cutting time, and break and working time on the total cutting time.

The main window of the software on the left shows the buttons to calculate the required tasks, and on the right, the list of names next to which are displayed the calculation results (Figure 2). By clicking on "Open file" the operator can select and upload the position file (.pos) obtained with the RTKLIB software. By clicking on "Create overall report", two or more position files can be pooled together.

Once the position file had been opened, a preliminary data processing consisted in the selection of the position file points that were classified as being of acceptable quality. The software then excluded all points with a variance higher than $0.05 \mathrm{~m}$ on the reconstruction of the ENU coordinates, then the resulted accuracy was $\pm 0.05 \mathrm{~m}$. Finally, the software only accepted the trajectories that travelled with a forward speed higher than $0.005 \mathrm{~m} \mathrm{~s}^{-1}$ and lower than $1.7 \mathrm{~m} \mathrm{~s}^{-1}$. After the preliminary phase, the software shows the coordinates of the robot mower on a scaled two-dimensional map (Figure 3). From the screen an operator can then select the area of interest on the map by manually designing a perimeter along the external coordinates of the robot mower (external red triangles in Figure 3). Once the area of interest has been selected, by setting the time and the length of the robot mower cut, and clicking on "Show cut", the turfgrass cut area by the robot mower at a desired time is graphically shown on the screen (Figure 4). By clicking on "Hide cut", the cut area is hidden. By clicking on "Calculate cover", the percentage of cut is reported on the screen. At the same time the distance travelled by the robot is also shown (Figure 4). By clicking on "Calculate intersections" and "Calculated speed", the number intersections of the robot mower trajectories and the mean forward speed were displayed (Figure 4).

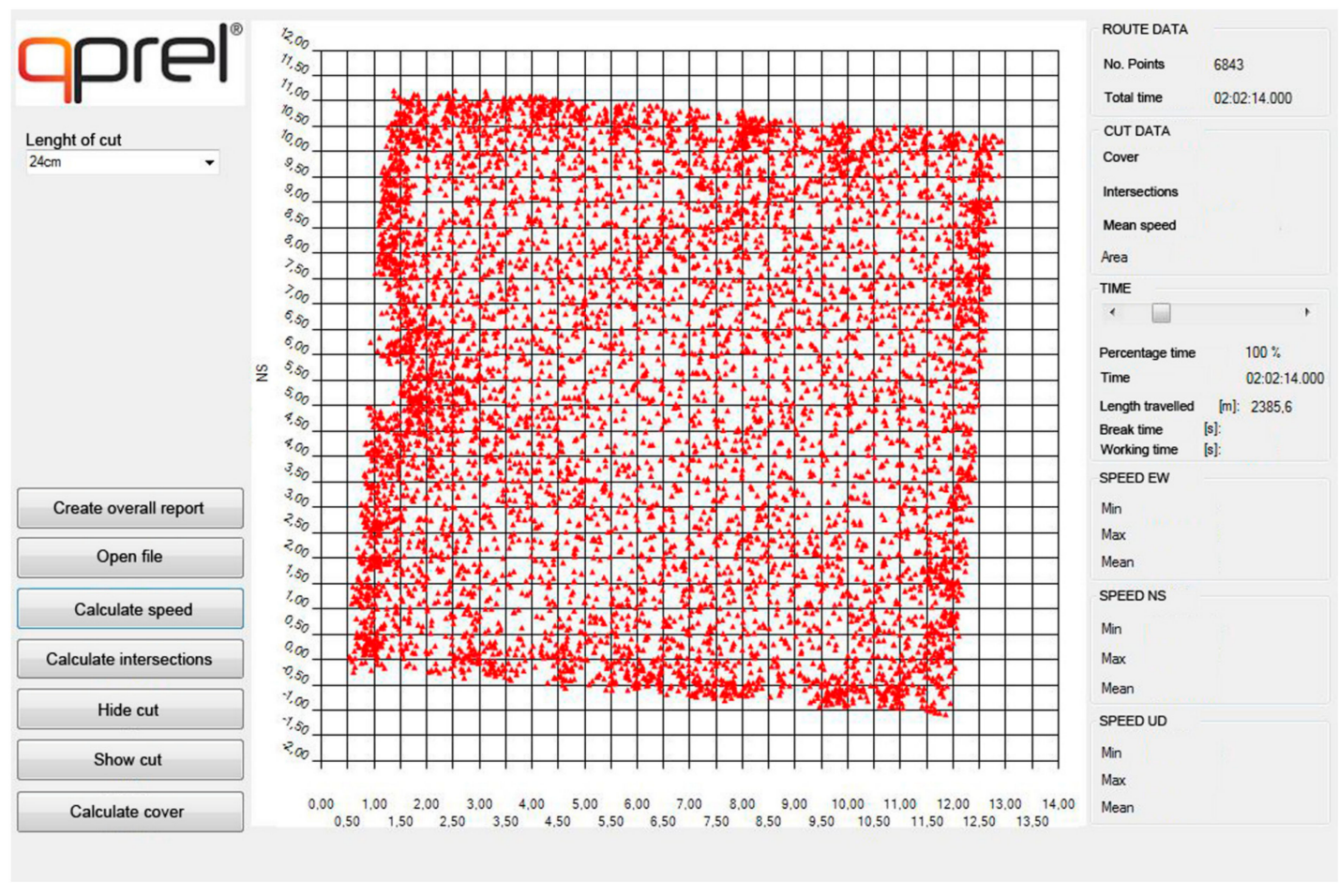

Figure 3. Window of the "Robot mower tracking data calculator" software showing the robot mower coordinates (red triangles) within a square turfgrass area shape for the total cutting time (two hours and two minutes). 


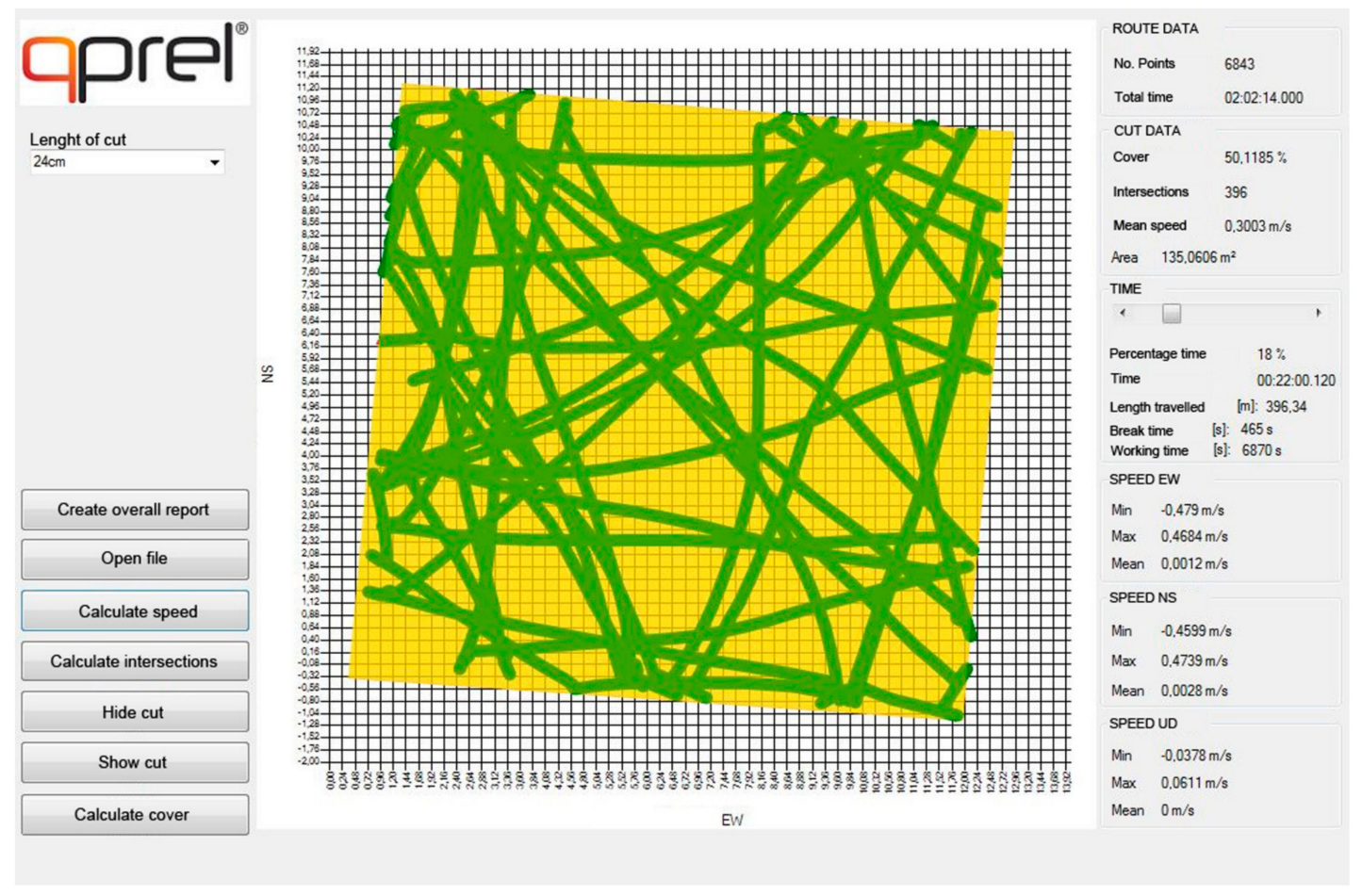

Figure 4. Window of the "Robot mower tracking data calculator" software showing the $50 \%$ of turfgrass cut area by the robot mower after 22 min of work (green lines). The yellow area is the area corresponding to $135 \mathrm{~m}^{2}$. The mean speed in the CUT DATA panel is the ratio between the distance travelled and time elapsed. The SPEED NS EW and UD are the north to south, east to west, and up to down speeds, considered as vectors with their magnitude and direction. The zero point of the axis is the base station position.

\subsection{Experimental Set Up, Design, and Treatments}

The experiment was conducted in autumn 2017 at the experimental farm of the Department of Agriculture, Food and Environment of the University of Pisa (San Piero a Grado, Pisa, Italy; $43^{\circ} 40^{\prime}$ $\mathrm{N}, 10^{\circ} 19^{\prime} \mathrm{E}, 6 \mathrm{~m}$. a.s.l.) on a mature warm-season turfgrass stand of Manila grass (Zoysia matrella (L.) Merr., cv 'Zeon'). The turf was in an open area, to avoid inaccurate position estimation results with the RTK-GNSS in a residential area occluded by walls and tree canopies.

The robot mower used was a Husqvarna Automower ${ }^{\circledR} 330 \mathrm{X}$ updated to 430X [35]. It moves randomly by travelling along straight lines and turned when it intercepts the boundary wire [36]. The robot was left to cut in different turfgrass area shapes: A square (side $=11.62 \mathrm{~m}$ ), a rectangle ( short side $=4.80 \mathrm{~m}$, long side $=28.10 \mathrm{~m})$, a right-angled triangle $($ base $=28.00 \mathrm{~m}$, height $=9.65 \mathrm{~m})$, and a circle (radius $=6.56 \mathrm{~m}$; Figure 5 ). These four shapes were obtained by manually positioning the boundary wire. The total area of each shape was $135 \mathrm{~m}^{2}$, which corresponded to the area capacity per hour stated in the manufacturer's manual [36]. The areas were devoid of internal obstacles. The robot mower cut continuously for two hours, without stopping to recharge the battery. The base station was left in the same position throughout the experiment. The cut was replicated four times for each shape, with a total of 16 cuts. 


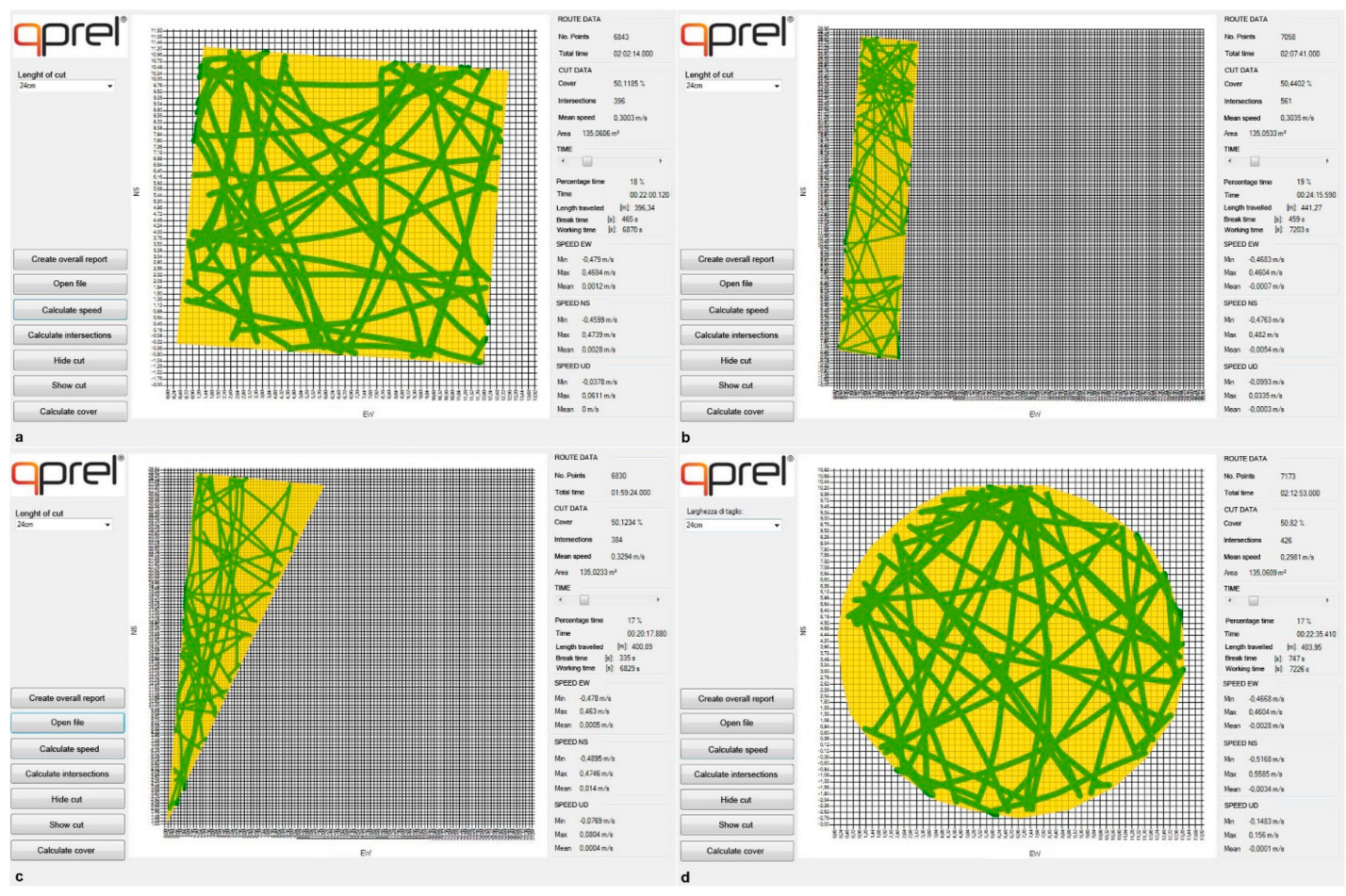

Figure 5. Screenshots of the software "Robot mower tracking data calculator" showing the different turfgrass area shapes with an area of $135 \mathrm{~m}^{2}$ selected (yellow area shapes): (a) Square, (b) rectangle, (c) triangle, and (d) circle. In these screenshots the percentage of cut area was 50\% (green lines).

\subsection{Data Collection}

The distance travelled by the robot, the mean forward speed, the intersections of the robot mower trajectories, and the turfgrass cut area percentage data were extracted from the software. The mean forward speed was extracted two hours after cutting. For the other variables, the data were extracted from the software every $15 \mathrm{~min}$ for a total time of two hours, in order to have nine time points for four replicates of the experiment in each turfgrass area shape. Other important data, such as the number of turnings and turning time, were taken manually because the software is not able to extract them. The number of turnings was counted 12 times (replicates) for $10 \mathrm{~min}$ in each area shape, and then was reported as the number of turnings in $120 \mathrm{~min}$. The turning time was measured with a stopwatch 40 times for each shape. The efficiency of the robot mower was calculated by dividing the real cut area in $135 \mathrm{~m}^{2}$ by the potential cut area that the robot would cut if it went straight without turnings. The efficiency thus calculated gave an idea of the cut overlapping within the $135 \mathrm{~m}^{2}$ turfgrass area. The efficiency was computed every $15 \mathrm{~min}$.

\subsection{Statistical Analysis}

Data normality was established using the Shapiro-Wilk test. Other tests consisted of Student's $t$-test to verify that the mean of the errors was not significantly different from zero, the Breusch-Pagan for homoscedasticity, and the Durbin-Watson test for serial correlation.

The distance travelled by the robot and the robot mower efficiency data were modeled in a linear mixed model in R software [37], using the extension package 'ImerTest' (tests in linear mixed effects models) [38]. The number of intersections of the robot mower trajectories was modeled in a generalized linear mixed model in R software, using the extension package 'Ime4' (fitting linear mixed-effects models using lme4) [39] and setting the Poisson distribution. The model conducted a log transformation. For these models a combined variable/factor was constructed from the time and shape of the turfgrass area factors, which was the fixed factor. The random factor was the interaction between the shape of the turfgrass area and the replicates. 
The mean forward speed at $120 \mathrm{~min}$ from the start of the cutting and the turning time were modeled in a linear mixed model in R software using the extension package 'lmerTest'. The number of turnings in 120 min of cutting data was modeled in a generalized linear mixed model in R software, using the extension package 'Ime4' and setting the Poisson distribution. The model conducted a log transformation. For these models the fixed factor was the shape of the turfgrass area, and the random factor was the replicates.

An analysis of variance was run for each model. The extension package 'ggplot2' (elegant graphics for data analysis) [40] was used to plot graphs.

The turfgrass cut area was modeled in a two-stage meta-analysis dose-response model using the extension package 'medrc' [41]. The meta-analysis strategy is a simple and robust method to summarize effective times of cutting estimates [42]. Time was the independent variable. The interaction between the shape of the turfgrass area and the replicates was the factor identifying individual curves. The shape of the turfgrass area was the factor containing the between-curve grouping of the data. The non-linear function was a two-parameter asymptotic regression:

$$
f(x)=d\left(1-\exp \left(-\frac{x}{e}\right)\right)
$$

The parameter $\mathrm{d}$ is the upper limit (for $x$ going to infinity) and the parameter e $>0$ determines the steepness of the increase as $x$ [43]. A simpler model was used to show trends graphically using the 'drc' package because the extension package 'medrc' was under development and did not draw the graph at the moment [44].

\section{Results}

\subsection{Distance Travelled by the Robot, Number of Turnings, Turning Time, and Mean Forward Speed}

The distance travelled by the robot mower was affected by the interaction between the time and the shape of the turfgrass area $(p$-value $<0.001)$. Means and $95 \%$ confidence intervals are reported in Figure 6 . The distance travelled in all the different shaped areas was significantly similar for one hour. At $75 \mathrm{~min}$ from the start of the cutting, the distance travelled in the triangle and square shapes was higher than in the circle. Between $90 \mathrm{~min}$ to $120 \mathrm{~min}$ after the start of the cutting, the distance travelled in the triangle and square was higher than both the circle and rectangle.

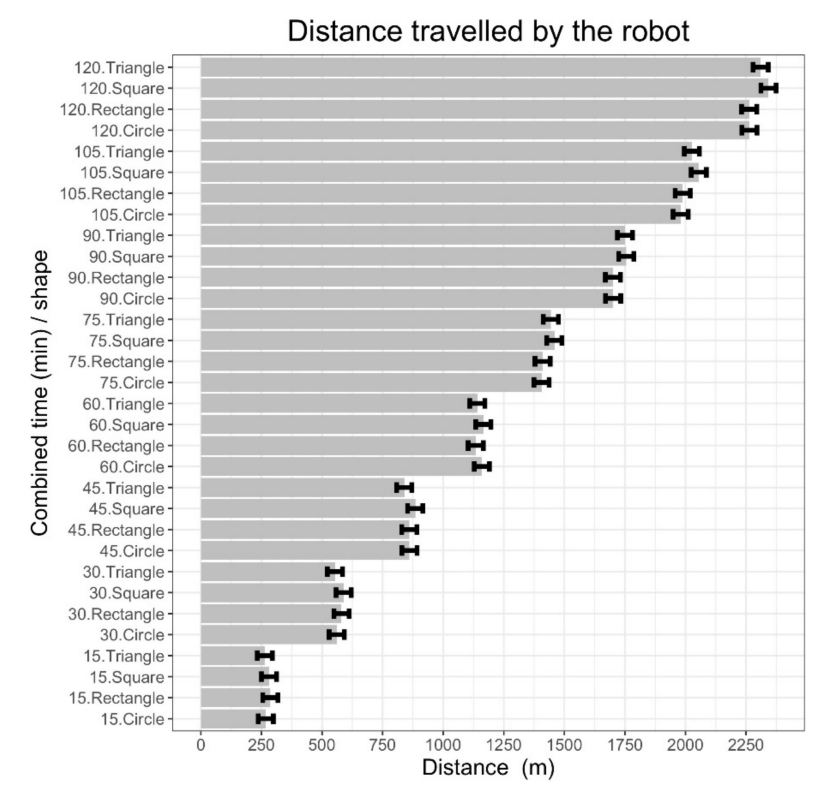

Figure 6. Means and 95\% confidence intervals of the distance travelled by the robot mower in the four turfgrass areas (square, rectangle, triangle, and circle) at 15, 30, 45, 60, 75, 90, 105, and 120 min after the start of cutting. 
The number of turnings was affected by the shape of the turfgrass area ( $p$-value $<0.001)$. The inverse transformed mean number of turnings in 120 min of cutting was 404 (lower $=393$, upper $=416)$ in the rectangle, 357 (lower $=347$, upper $=368)$ in the triangle, 343 (lower $=333$, upper $=354$ ) in the square, and 324 (lower $=314$, upper $=335$ ) in the circle. The rectangular shape showed the highest statistically significantly number of turnings, and the circle, the lowest. In the square and triangle shapes, the number of turnings was statistically similar.

The turning time was affected by the shape of the turfgrass area $(<0.001)$. The mean turning time was $6.28 \mathrm{~s}( \pm 0.16 \mathrm{~s}), 5.45 \mathrm{~s}( \pm 0.16 \mathrm{~s}), 5.42 \mathrm{~s}( \pm 0.16 \mathrm{~s})$, and $5.30 \mathrm{~s}( \pm 0.16 \mathrm{~s})$ in the circle, rectangle, triangle, and square, respectively. In the circle, the turning time was the highest. In the other shapes the turning time was statistically similar.

The mean forward speed of the robot mower at $120 \mathrm{~min}$ after the start of the cutting was affected by the shape of the turfgrass area ( $p$-value $=0.046$ ). The mean forward speed was $0.315 \mathrm{~m} \mathrm{~s}^{-1}, 0.316 \mathrm{~m}$ $\mathrm{s}^{-1}\left( \pm 0.003 \mathrm{~m} \mathrm{~s}^{-1}\right), 0.320 \mathrm{~m} \mathrm{~s}^{-1}\left( \pm 0.003 \mathrm{~m} \mathrm{~s}^{-1}\right)$, and $0.324 \mathrm{~m} \mathrm{~s}^{-1}\left( \pm 0.003 \mathrm{~m} \mathrm{~s}^{-1}\right)$ in the rectangle, circle, triangle, and square, respectively. Only in the square was the forward speed statistically higher than in the rectangle and circle. Other pair-wise comparisons were statistically similar.

\subsection{Intersections of the Robot Mower Trajectories}

The number of intersections of the robot mower trajectories was affected by the interaction between the time and the shape of the turfgrass area ( $p$-value $\leq 0.001)$. Inverse transformed means and $95 \%$ confidence intervals are reported in Figure 7 . The intersections of the trajectories in the rectangle, which 15 min after the start of the cutting were statistically significantly higher than in the other shapes, between $75 \mathrm{~min}$ to $120 \mathrm{~min}$ after the start of the cutting were statistically significantly lower than in the other shapes. After $105 \mathrm{~min}$ from the start of the cutting, the intersections were statistically significantly higher in the square than in the other shapes. In the triangle and circle, the number of intersections was similar for most of the cutting time.

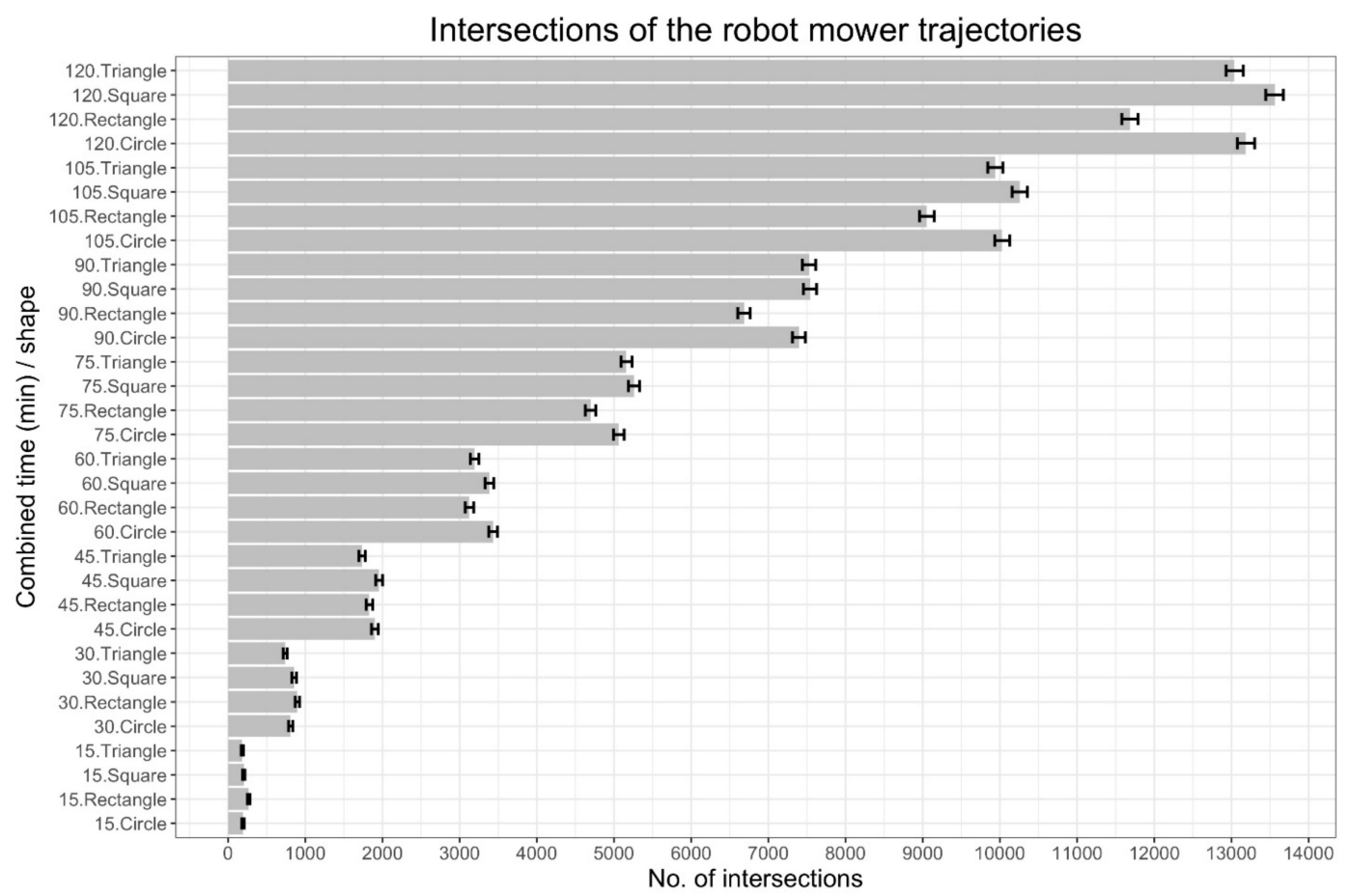

Figure 7. Inverse-transformed means and $95 \%$ confidence intervals of the number of intersections of the robot mower trajectories in the different turfgrass area shapes (square, rectangle, triangle, and circle) at $15,30,45,60,75,90,105$, and $120 \mathrm{~min}$ from the start of the cutting. 


\subsection{Area of the Turfgrass Cut}

The lack-of-fit test $(p$-value $=0.988)$ indicated that the asymptotic regression model was comparable to a more general ANOVA model using an approximate F test. The parameters of the non-linear asymptotic regressions (Equation (1)) and effective cutting times estimated from the two-stage meta-analysis dose-response model are reported in Table 1. The data trend is shown in Figure 8. In the rectangle $10 \%, 50 \%, 85 \%$, and $90 \%$ of the area cut was reached slower than in the triangle and circle ( $p$-values $=0.02$ and 0.03 , respectively), and in a similar time as the square ( $p$-value $=0.12)$. In the triangle, circle, and square, $10 \%, 50 \%, 85 \%$, and $90 \%$ of the cut area was reached in a significantly similar time ( $p$-values $=0.32,0.60$, and 0.61 , respectively). After two hours of cutting, there were no statistically differences between the percentage turfgrass area cut in the different shapes (Table 1 , $\mathrm{d}$ parameter). This suggests that after 120 min of cutting, the shape of the turfgrass did not affect the percentage cut.

Table 1. Parameters of the non-linear asymptotic regressions (Equation (1)) and effective times of cut estimated from the two-stage meta-analysis dose-response model (Figure 8 is a simplified graphical representation because the package 'medrc' does not draw the graph at the moment).

\begin{tabular}{lcccccc}
\hline Shape & \multicolumn{5}{c}{ Effective Times-ET (min) } \\
\hline \multicolumn{1}{c}{$\mathbf{d}$} & $\mathbf{e}$ & $\mathbf{E T}_{\mathbf{1 0}}$ & $\mathbf{E T}_{\mathbf{5 0}}$ & $\mathbf{E T}_{\mathbf{8 5}}$ & $\mathbf{E T}_{\mathbf{9 0}}$ \\
\hline Square & $98.363(1.166)$ & $31.398(1.561)$ & $3.308(0.164)$ & $21.763(1.082)$ & $59.565(2.962)$ & $72.296(3.595)$ \\
Rectangle & $98.018(1.198)$ & $35.153(1.586)$ & $3.704(0.167)$ & $24.367(1.100)$ & $66.690(3.009)$ & $80.944(3.652)$ \\
Triangle & $98.092(1.216)$ & $29.052(1.597)$ & $3.061(0.168)$ & $20.137(1.107)$ & $55.115(3.029)$ & $66.894(3.676)$ \\
Circle & $98.872(1.186)$ & $30.226(1.576)$ & $3.185(0.166)$ & $20.951(1.092)$ & $57.342(2.989)$ & $69.597(3.628)$ \\
\hline
\end{tabular}

$\mathrm{d}$ : Is the upper limit of the curve (for time go to infinity); e: Determines the steepness of the increase as time; $\mathrm{ET}_{10}$ : Is the time required to cut $10 \%$ of the turfgrass area; $\mathrm{ET}_{50}$ : Is the time required to cut $50 \%$ of the turfgrass area; $\mathrm{ET}_{85}$ : Is the time required to cut $85 \%$ of the turfgrass area; $\mathrm{ET}_{90}$ : Is the time required to cut $90 \%$ of the turfgrass area.

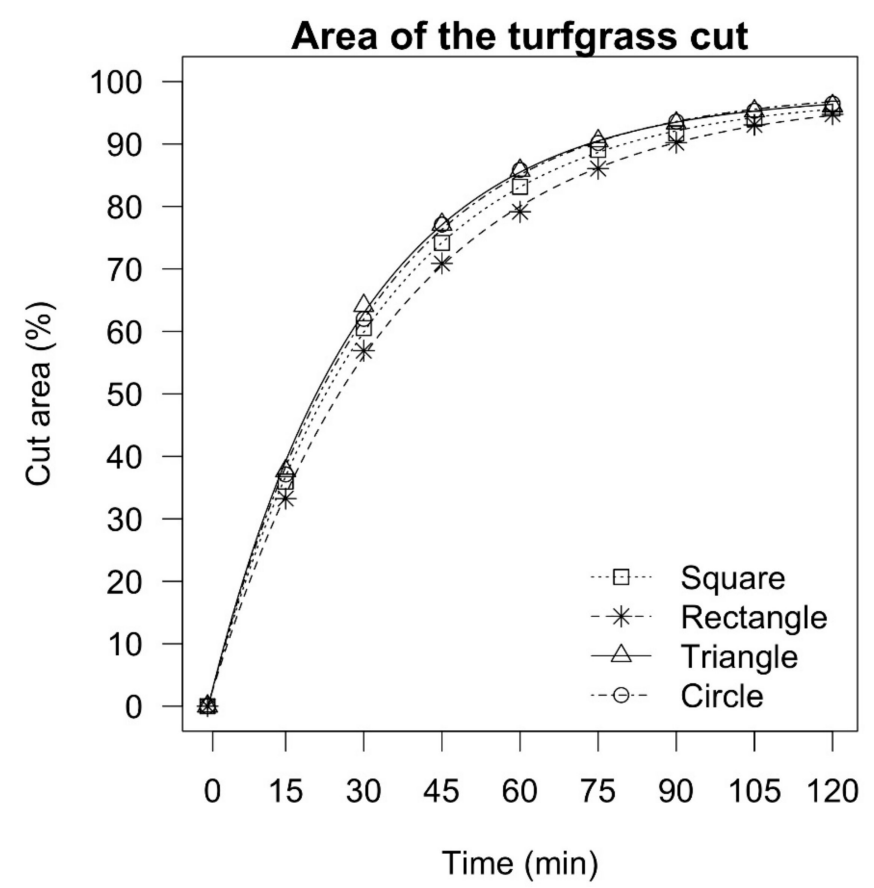

Figure 8. Fitted asymptotic regression curves with averages points of the cut area as a function of the time ( $\mathrm{min}$ ) and the shape of the turfgrass area. This graph is the result of a simplified model (which follows a non-linear two-parameter asymptotic regression but not the two-stage meta-analysis dose-response model), because the package 'medrc' of $\mathrm{R}$ [41] used for the analysis was not yet programmed to graph plots. 


\subsection{Robot Mower Efficiency}

The efficiency of the robot mower was affected by the interaction between the time and the shape of the turfgrass area $(p$-value $<0.001)$. Means and $95 \%$ confidence intervals are reported in Figure 9. After $15 \mathrm{~min}$ of cutting the efficiency of the robot mower in the triangle and circle was statistically similar, and significantly higher than in the square and rectangle. For this lapse of time, the robot mower showed the lowest efficiency in the rectangular shape. After $30 \mathrm{~min}$ of cutting, the robot mower efficiency was significantly different in all the shapes, with the highest value was found for the triangle $(65 \% \pm 1 \%)$, followed by the circle $(62 \% \pm 1 \%)$, square $(58 \% \pm 1 \%)$, and rectangle $(55 \% \pm 1 \%)$. After $45 \mathrm{~min}$, the robot mower efficiency in the triangle and circle was again statistically similar. After this time, the efficiency in the square and rectangle was significantly similar, and lower than in the triangle and circle. After an hour of cutting, the efficiency in the triangle was higher than in the rectangle, but similar to the other shapes. On the other hand, the efficiency in the square, circle, and rectangle was significantly similar. After $75 \mathrm{~min}$ of cutting, the robot mower efficiency was significantly similar in all the shapes, which was maintained until two hours of cutting.

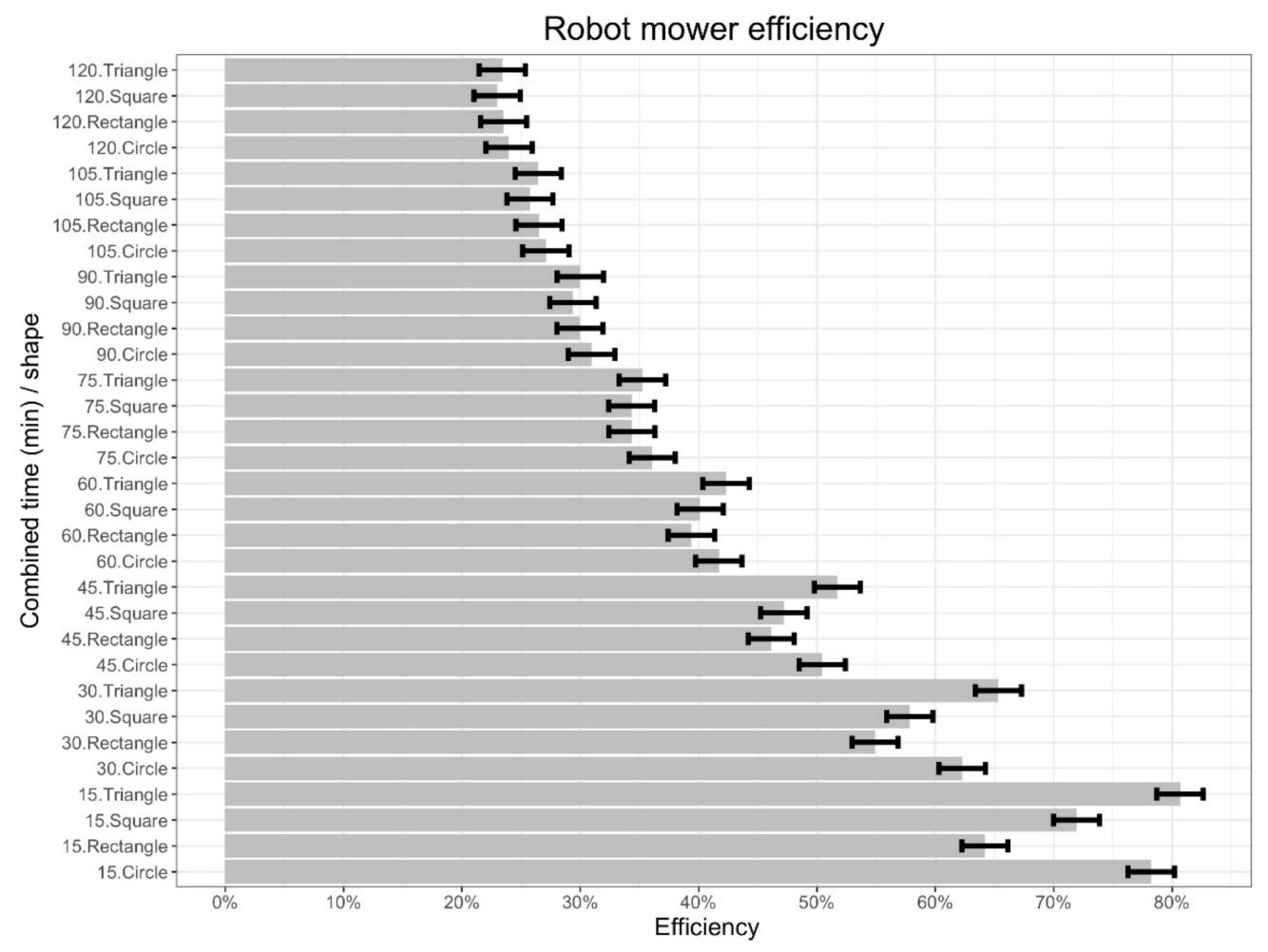

Figure 9. Means and $95 \%$ confidence intervals of the efficiency of the robot mower in the four turfgrass area shapes at $15,30,45,60,75,90,105$, and $120 \mathrm{~min}$ from the start of the cutting.

\section{Discussion and Conclusions}

The software extracted data on the distance travelled by the robot mower, the intersections of the trajectories, the mean forward speed, the differently shaped cut areas of turfgrass, and the derived efficiency data. These data, without the use of a remote system and the software to acquire, calculate, and extract data, would have been very difficult to collect manually.

Data processing led to interesting information on the functioning of the robot mower in the four turfgrass shapes. The distance travelled by the robot mower after two hours of cutting was highest in the triangle and square. This was due to the greater number of turnings in the rectangle and the longer turning time for the circle, which led to a significantly similar mean forward speed. The highest number of turnings in the rectangle was probably due to the shorter side of this shape 
$(4.8 \mathrm{~m})$, which increased the probability that the robot encountered the boundary wire compared with the other shapes. The highest turning time in the circle was probably due to the geometry of the boundary wire, which not being a straight line, forced the robot mower to execute a higher degree of turning, which increased the turning time. In fact, the degree of turning of robot mowers is random and is different depending on the boundary wire geometry [45]. In the triangle and square area, the number of turnings, the turning time, and the mean forward speed were similar.

The intersections of the robot mower trajectories were related to the distance travelled and probably to where the turning occurred on the boundary of the shape. It was possible that in the rectangle the narrow and long shape enabled trajectories to be designed that from $0 \mathrm{~min}$ to $15 \mathrm{~min}$ of cutting had more crossing lines compared with other shapes, and from $75 \mathrm{~min}$ to $120 \mathrm{~min}$ had fewer crossing lines. In the square, the position of the turnings led to the highest number of crossed lines from $105 \mathrm{~min}$ to $120 \mathrm{~min}$ of cutting. The positions of the turnings in the circle and triangle shapes probably led to a similar number of crossed lines.

In the rectangle, the robot mower was slower to reach the same percentages of cut areas as in the other shapes. In the triangle, square, and circle, $85 \%$ of the cut area was reached after about an hour of cutting, whereas in the rectangle, this percentage was reached a few minutes later. In any case, in all of the shapes, an average of $98 \%$ of the cut areas were reached after two hours (parameter d, Table 1). This did not contradict the manufacturer's manual, which stated a range from $80 \%$ to $120 \%$ of cut area after one hour of cutting [36].

Data extracted from the software and subsequently analyzed, gave the parameters (Table 1) needed for an accurate estimation of the percentage of cut turfgrass as a function of time (Equation (1)). For example, by substituting the mean values of parameters $d$ and e in Equation (1), the percentage of cut area after one hour of cutting was $80.2 \%$ in the rectangle, $83.8 \%$ in the square, $85.3 \%$ in the circle, and $85.7 \%$ in the triangle.

In the experimental area of $135 \mathrm{~m}^{2}$, an average of $98 \%$ of cut surface (parameter d, Table 1) was obtained after two hours of continuous cutting (without stopping to recharge the battery) in all the shapes. Probably, in a turfgrass area larger than $135 \mathrm{~m}^{2}$, an average of $135 \mathrm{~m}^{2}$ of cut area could be obtained in a shorter time than two hours, because the overlapping would have been lower. In this experiment, after $75 \mathrm{~min}$ of cutting, the efficiency was about $35 \%$ in all the shapes, thus indicating a high level of overlapping. The efficiency, which represents an indicator of overlapping within the cutting area, decreased by increasing the time, which after two hours of cutting was about $25 \%$. At this time the overlapping was maximum in all the shapes.

The effect of the shape on the efficiency was observed in the first hour of cutting, suggesting that the shape determined the number of overlaps for this period of time. From 75 min to $120 \mathrm{~min}$ of cutting, the efficiency was similar in all the shapes, suggesting that the random path planning tended to homogenize the overlaps as the time increased, irrespectively of the shape of the turfgrass. However, the robot mower tracking data system is currently not able to unequivocally calculate the percentage of overlaps, nor how many times the overlaps occurred in each area shape.

Ideally, a robot mower should cover the area in the most efficient way possible, saving energy and time [19]. The efficiency of the robot mower could be improved by planning a coverage path that allows the robot to follow the shortest route rather than moving at random [46]. Bosse et al. [19] tested a prototype robot mower with a working width of $2 \mathrm{~m}$ in a turfgrass area of $321 \mathrm{~m}^{2}$ and found that by using spiral inward (near the perimeter) and spiral shift (in the inner area) path planning algorithms, $95 \%$ of the area was covered in $15 \mathrm{~min}$. However, the working width of this prototype was about eight-fold larger than commercial robot mowers, which reduced the time needed to cover the whole turfgrass area compared with the robot mower used in this experiment, which had a working width of $0.24 \mathrm{~m}$.

The integration of the robot mowers with RTK-GNSS guidance systems would facilitate a pre-planned path in order to optimally and efficiently cover the area of interest [21]. However, the inaccurate functioning of GNSS devices near buildings and tree canopies in residential areas, where turfgrasses are often located, 
remains a problem. A sensor fusion (ultrasonic sensor, laser rangefinder, LIDAR, GNSS, odometry, cameras, stereocam, etc.) is thus needed $[14,24]$, which would inevitably increase the cost of robot mowers.

In conclusion, the system used for tracking the robot mower and the software for calculating and extracting information provided a simple way of obtaining useful data on the performance of a common commercial robot mower working on different turfgrass area shapes. The results of the data analysis could be used by manufacturers, entrepreneurs, and practitioners to facilitate their decision-making, reduce costs, and improve the quality of the product.

The software could be enhanced to also extract data on the number of turnings and turning time. In addition, an algorithm to estimate the percentage of overlapping could be integrated. Future research could focus on the integration of the robot mower with a guidance system based on the tracking data system developed in this paper. Data regarding the boundary of the turfgrass could be tracked and then an appropriate pre-planning path map could be created to allow the robot mower to cover the whole cut area in the least possible time. However, the problem of the majority of turfgrass areas being located in residential areas near to buildings and trees canopies, which reduces the precision of GNSSs receivers, still needs to be resolved.

Author Contributions: Conceptualization, M.F. and S.P.; methodology, M.F., S.P., L.M., C.F., L.C., M.G., N.G., S.M. and M.P.; validation, M.F., S.P. and L.M.; formal analysis, L.M., M.F. and S.P.; investigation, M.F., L.M. and S.P.; resources, M.F., L.M., C.F., L.C., M.G., N.G., S.M. and M.P.; software development, S.P. and M.F.; data curation, L.M.; writing—original draft preparation, L.M. and M.F.; writing—review and editing, L.M., M.F. and S.P.; visualization, M.F., L.M., S.P., C.F., L.C., M.G., N.G., S.M., M.R., A.P., M.V. and M.P.; supervision, M.F., A.P., M.R., M.V.; project administration, A.P. and M.V.

Funding: This study was part of the "Tecnologie innovative per una gestione efficiente ed eco-compatibile dei tappeti erbosi" project, funded by the University of Pisa (Progetti di Ricerca di Ateneo, 2016).

Acknowledgments: This study was part of the "Tecnologie innovative per una gestione efficiente ed eco-compatibile dei tappeti erbosi" project, funded by the University of Pisa (Progetti di Ricerca di Ateneo, 2016). The authors would like to thank the Master's student Ilaria Duranti of the Department of Agriculture, Food and Environment of University of Pisa (Pisa, Italy) for her significant contribution to the experiment. The authors also wish to thank Christian Ritz (Department of Nutrition, Exercise and Sports, University of Copenhagen) for building the two-stage meta-analysis dose-response model using the extension package 'medrc' of $R$, which is still under development, and for his support in the statistical analysis. The authors also wish to thank Qprel ${ }^{\circledR}$ srl (Qualità nella PRogettazione ELettronica. http://qprel.com) (Pistoia, Italy), and FERCAD S.p.a. (Altavilla Vicentina, Vicenza, Italy) for providing the robot mower used in this experiment and for their support.

Conflicts of Interest: The authors declare no conflict of interest.

\section{References}

1. Tseng, C.; Jiang, J.; Lee, R.; Lu, F.; Ouyang, C.; Chen, Y.; Chang, C. Feasibility study on application of GSM-SMS technology to field data acquisition. Comput. Electron. Agric. 2006, 53, 45-59. [CrossRef]

2. Hejazian, M.; Hosseini, S.; Lotfalian, M.; Ahmadikoolaei, P. Possibility of global positioning system (GPS) application for time studies in forest machinery. Eur. J. Exp. Biol. 2013, 3, 93-98.

3. Sarri, D.; Martelloni, L.; Vieri, M. Development of a prototype of telemetry system for monitoring the spraying operation in vineyards. Comput. Electron. Agric. 2017, 142, 248-259. [CrossRef]

4. Abeledo, M.C.; Bruschetti, F.; Priano, D.A.; Calbosa, D.; Crubellier, R.; Iriso, P.; Abete, E. Application of wireless technology to determine optimum maturity in strains of Malbec vineyards for Argentine wine sectors. In Congreso Aergentino de Ciencias de la Informatica y Desarrollos de Investigacion (CACIDI 2016).; Institute of Electrical and Electronics Engineers Inc.: Buenos Aires, Argentina, 2016; 7785984. [CrossRef]

5. Ho, V.; Rauf, K.; Passchier, I.; Rijks, F.; Witsenboer, T. Accuracy assessment of RTK GNSS based positioning systems for automated driving. In Proceedings of the 5th Workshop on Positioning, Navigation and Communications (WPNC 2018), Campus Center/IRC. Bremen, Germany, 25-26 October 2018; p. 8555805.

6. Mohamed, A.; Ren, J.; Lang, H.; El-Gindy, M. Optimal path planning for an autonomous articulated vehicle with two trailers. Int. J. Autom. Control. 2018, 12, 449-465. [CrossRef] 
7. Hou, K.; Zhang, Y.; Shi, J.; Zheng, Y. Motion planning based on artificial potential field for unmanned tractor in Farmland. In Advances in Human Factors in Robots and Unmanned Systems; Springer: Cham, Switzerland; Orlando, FL, USA, 2018; pp. 153-162.

8. Alipour, K.; Robat, A.B.; Tarvirdizadeh, B. Dynamics modeling and sliding mode control of tractor-trailer wheeled mobile robots subject to wheels slip. Mech. Machin. Theor. 2019, 138, 16-37. [CrossRef]

9. Kassaeiyan, P.; Tarvirdizadeh, B.; Alipour, K. Control of tractor-trailer wheeled robots considering self-collision effect and actuator saturation limitations. Mech. Syst. Signal Proc. 2019, 127, 388-411. [CrossRef]

10. Sun, Q.C.; Odolinski, R.; Xia, J.C.; Foster, J.; Falkmerc, T.; Lee, H. Validating the efficacy of GPS tracking vehicle movement for driving behaviour assessment. Travel Behav. Soci. 2017, 6, 32-43. [CrossRef]

11. Huang, P.; Zhang, Z.; Luo, X.; Zhang, J.; Huang, P. Path Tracking Control of a Differential-Drive Tracked Robot Based on Look-ahead Distance. IFAC-PapersOnLine 2018, 51, 112-117. [CrossRef]

12. El-Mowafy, A.; Kubo, N. Integrity monitoring of vehicle positioning in urban environment using RTK-GNSS, IMU and speedometer. Meas. Sci. Technol. 2017, 28, 055102. [CrossRef]

13. Sun, Q.C.; Xia, J.C.; Foster, J.; Falkmer, T.; Lee, H. Pursuing Precise Vehicle Movement Trajectory in Urban Residential Area Using Multi-GNSS RTK Tracking. Transport. Res. Proced. 2017, 25, 2356-2372. [CrossRef]

14. de Paula Veronese, L.; Badue, C.; Auat Cheein, F.; Guivant, J.; De Souza, A.F. A single sensor system for mapping in GNSS-denied environments. Cognit. Syst. Res. 2019, 56, 246-261. [CrossRef]

15. De Saxe, C.; Cebon, D. Estimation of trailer off-tracking using visual odometry. Vehicle System Dynamics. 2019, 57, 752-776. [CrossRef]

16. Chiodini, S.; Pertile, M.; Giubilato, R.; Salvioli, F.; Barrera, M.; Franceschetti, P.; Debei, S. Experimental evaluation of a camera rig extrinsic calibration method based on retro-reflective markers detection. Measur. J. Int. Measur. Conf. 2019, 140, 47-55. [CrossRef]

17. Zaman, S.; Comba, L.; Biglia, A.; Ricauda Aimonino, D.; Barge, P.; Gay, P. Cost-effective visual odometry system for vehicle motion control in agricultural environments. Comput Electron Agric. 2019, 162, 82-94. [CrossRef]

18. Galceran, E.; Carreras, M. A survey on coverage path planning for robotics. Rob. Auton. Syst. 2013, 61, $1258-1276$. [CrossRef]

19. Bosse, M.; Nourani-Vatani, N.; Roberts, J. Coverage Algorithms for an Under-actuated Car-Like Vehicle in an Uncertain Environment. In Proceedings of the IEEE International Conference on Robotics and Automation, Roma, Italy, 10-14 April 2007; pp. 698-703.

20. Ahmad, N.; bin Lokman, N.; Wahab, M.H.A. Autonomous Lawnmower Using FPGA implementation. In Proceedings of the International Engineering Research and Innovation Symposium (IRIS). IOP Conference Series: Materials Science and Engineering, Melaka, Malaysia, 24-25 November 2016; The R Foundation: Vienna, Austria, 2016; pp. 1-8. [CrossRef]

21. Melita, C.D.; Muscato, G.; Poncelet, M. A Simulation Environment for an augmented global navigation satellite system assisted autonomous robotic lawn-mower. J. Intell. Robot Syst. 2013, 71, 127-142. [CrossRef]

22. Zhou, J.; Zhu, Y.; Yang, C. Path Planning and Trajectory Tracking Control of Large Intelligent Mowing Robot Based on GPS-RTK. Int. J. Circuits Syst. Signal Proc. 2019, 13, 132-139.

23. Ambrogio Robot. L400 ELITE. Available online: https://www.ambrogiorobot.com/en/models/view/1400-elite (accessed on 21 March 2019).

24. Dalecký, S.; Snášelová, P.; Luźa, R.; Žák, M.; Rozman, J.; Zbořil, F.V. SmartMowers as a part of a SmartCity. In Proceedings of the International Carpathian Control Conference (ICCC), Tatranská Lomnica, Slovak Republic, 29 May-1 June; pp. 156-160.

25. Raja, P.; Pugazhenthi, S. Optimal path planning of mobile robots: A review. Int. J. Phys. Sci. 2012, 9, 1314-1320.

26. Li, Z.; Zhang, T.; Qi, F.; Tang, H.; Niu, X. Carrier phase prediction method for GNSS precise positioning in challenging environment. Adv. Space Res. 2019, 637, 2164-2174. [CrossRef]

27. Emlid. Reach RTK docs. Specification. Available online: https://docs.emlid.com/reach/specs/ (accessed on 21 March 2019).

28. ublox. NEO/LEA-M8T series. Available online: https://www.u-blox.com/en/product/neolea-m8t-series (accessed on 21 March 2019).

29. Intel Software. Intel®Edison Development Platform. Available online: https://software.intel.com/en-us/ node/737243 (accessed on 21 March 2019). 
30. Takasu, T. RTKLIB ver. 2.4.2 Manual. Available online: http://www.rtklib.com/prog/manual_2.4.2.pdf (accessed on 21 March 2019).

31. Zyxel. NBG-418N v2. Wireless Router e Access Point N 300Mbps. Available online: http://www.zyxel.it/ Prodotti/Scheda/ZYXNBG-418NV2 (accessed on 21 March 2019).

32. Emlid. ReachView. Available online: https://docs.emlid.com/reach/common/reachview/ (accessed on 21 March 2019).

33. Qprel. Qualità nella PRogettazione ELettronica. Available online: http://qprel.com (accessed on 21 March 2019). (In Italian).

34. Microsoft. Install Visual Studio 2015. Available online: https://msdn.microsoft.com/en-us/library/e2h7fzkw. aspx (accessed on 21 March 2019).

35. Husqvarna. Husqvarna forest and garden products. Product guide-Robotic lawnmowers. Available online: https://www.husqvarna.com/us/catalogs/ (accessed on 21 March 2019).

36. Husqvarna. Operator's manual HUSQVARNA AUTOMOWER®420/430X/440/450X. Available online: http://service.webec.husqvarna.net/documents/HUSO/HUSO2017_NAen/HUSO2017_NAen_115875895.pdf (accessed on 21 March 2019).

37. R Core Team. R: A language and environment for statistical computing. R Foundation for Statistical Computing, Vienna, Austria. Available online: https://www.R-project.org/ (accessed on 21 March 2019).

38. Kuznetsova, A.; Brockhoff, P.B.; Christensen, R.H.B. lmerTest: Tests in Linear Mixed Effects Models. R package version 2.0-32. Available online: https://CRAN.R-project.org/package=lmerTest (accessed on 21 March 2019).

39. Bates, D.; Maechler, M.; Bolker, B.; Walker, S. Fitting Linear Mixed-Effects Models Using lme4. J. Stat. Softw. 2015, 67, 1-48. [CrossRef]

40. Wickham, H. ggplot2: Elegant Graphics for Data Analysis, 3rd ed.; Springer-Verlag: New York, NY, USA, 2009.

41. Gerhard, D.; Ritz, C. medrc: Mixed effect dose-response curves. R package version 1.1-0. Available online: https://rdrr.io/github/DoseResponse/medrc/man/metadrm.html (accessed on 21 March 2019).

42. Jiang, X.; Schneider, A.K. Summarizing EC50 estimates from multiple dose-response experiments: A comparison of a meta-analysis strategy to a mixed-effects model approach. Biom. J. 2014, 56, 493-512. [CrossRef] [PubMed]

43. Ritz, C. Asymptotic regression model. In Analysis of Dose-Response Curves; Ritz, C., Strebig, J.C., Eds.; 2016; pp. 7-8. Available online: https://cran.r-project.org/web/packages/drc/drc.pdf (accessed on 21 March 2019).

44. Ritz, C.; Baty, F.; Streibig, J.C.; Gerhard, D. Dose-Response Analysis Using R. PLoS ONE 2015, 10, e0146021. [CrossRef] [PubMed]

45. Tang, Q.; Schiehlen, W. From lawnmower dynamics to modelling, simulation and experiments of a differentially driven robot. In Advances on theory and practice of robots and manipulators; Ceccarelli, M., Glazunov, V.A., Eds.; Springer: Basel, Switzerland, 2014; pp. 365-374.

46. Wang, J.; Chen, J.; Cheng, S.; Xie, Y. Double heuristic optimization based on hierarchical partitioning for coverage path planning of robot mowers. In Proceedings of the 12th International Conference on Computational Intelligence and Security (CIS), Wuxi, Jiangsu Province, China, 16-19 December 2016; pp. 186-189. [CrossRef]

(C) 2019 by the authors. Licensee MDPI, Basel, Switzerland. This article is an open access article distributed under the terms and conditions of the Creative Commons Attribution (CC BY) license (http://creativecommons.org/licenses/by/4.0/). 